Revue internationale P.M.E.

Économie et gestion de la petite et moyenne entreprise

Revue

internationale

PME

\title{
Le financement de la Mittelstand allemande
}

\section{David B. Audretsch et Julie-Ann Elston}

Volume 8, numéro 3-4, 1995

URI : https://id.erudit.org/iderudit/1008361ar

DOI : https://doi.org/10.7202/1008361ar

Aller au sommaire du numéro

\section{Éditeur(s)}

Presses de l'Université du Québec

ISSN

0776-5436 (imprimé)

1918-9699 (numérique)

Découvrir la revue

Citer cet article

Audretsch, D. B. \& Elston, J.-A. (1995). Le financement de la Mittelstand allemande. Revue internationale P.M.E., 8(3-4), 121-147.

https://doi.org/10.7202/1008361ar

\section{Résumé de l'article}

Dans cet article, les auteurs cherchent à décrire comment la Mittelstand, c'est-à- dire les petites et moyennes entreprises, est financée en Allemagne. La première section rend compte du rôle de la Mittelstand allemande d'un point de vue statique et dynamique et établit des comparaisons avec les situations observables dans les principaux pays industriels développés. De manière générale, on s'aperçoit que la Mittelstand a joué un rôle plus important en Allemagne que dans des pays comme les États-Unis ou le Royaume-Uni. Le succès récurrent de la Mittelstand peut être expliqué par référence au système financier allemand qui se révèle largement relayé par des institutions connexes installées afin de satisfaire aux besoins spécifiques des grandes entreprises et des PME. Parallèlement, il est possible de prouver que malgré l'existence d'un tel système de financement, il existe une relation négative entre la taille des entreprises et la contrainte de liquidité qu'elles subissent. Il apparaît, enfin, que le système financier allemand se révèle particulièrement peu performant en ce qui concerne sa capacité à affecter des fonds aux entreprises naissantes dans les nouveaux secteurs d'activité.
Ce document est protégé par la loi sur le droit d'auteur. L’utilisation des services d’Érudit (y compris la reproduction) est assujettie à sa politique d'utilisation que vous pouvez consulter en ligne.

https://apropos.erudit.org/fr/usagers/politique-dutilisation/ 


\title{
Le financement de la Mittelstand ${ }^{1}$ allemande
}

\author{
David B. AUDRETSCH* \\ Julie-Ann ELSTON** \\ Wissenschaftszentrum Berlin für Sozialforschung
}

MOTS CLÉS

Finance - Allemagne - PME - Contrainte de liquidité

\begin{abstract}
RÉSUMÉ
Dans cet article, les auteurs cherchent à décrire comment la Mittelstand, c'est-àdire les petites et moyennes entreprises, est financée en Allemagne. La première section rend compte du rôle de la Mittelstand allemande d'un point de vue statique et dynamique et établit des comparaisons avec les situations observables dans les principaux pays industriels développés. De manière générale, on s'aperçoit que la Mittelstand a joué un rôle plus important en Allemagne que dans des pays comme les États-Unis ou le Royaume-Uni. Le succès récurrent de la Mittelstand peut être expliqué par référence au système financier allemand qui se révèle largement relayé par des institutions connexes installées afin de satisfaire aux besoins spécifiques des grandes entreprises et des PME. Parallèlement, il est possible de prouver que malgré l'existence d'un tel système de financement, il existe une relation négative entre la taille des entreprises et la contrainte de liquidité qu'elles subissent. II apparaît, enfin, que le système financier allemand se révèle particulièrement peu performant en ce qui concerne sa capacité à affecter des fonds aux entreprises naissantes dans les nouveaux secteurs d'activité.
\end{abstract}

* David B. Audretsch est professeur au Wissenschaftszentrum für Sozialforschung, dont il fut vice-directeur. Il est le fondateur et l'éditeur de la revue Small Business Economics : An International Journal. Il a publié plus d'une centaine d'articles et treize ouvrages dont le plus récent, intitulé Innovation and Industry Evolution, édité par les MIT Press en 1995. Adresse: WZB Reichpietschufer 50 10785, Berlin, Allemagne.

** Julie-Ann Elston est chargée de recherche au Wissenschaftszentrum für Sozialforschung. Elle a obtenu son doctorat à l'Université de Washington et met actuellement en œuvre un projet de recherche visant à procéder à la comparaison des systèmes de financement en Allemagne, aux États-Unis et au Japon. Adresse: WZB Reichpietschufer 50 10785, Berlin, Allemagne.

1. Ce terme désigne les entreprises dont le nombre de salariés est compris entre 200 et 500 ; étant donnée sa spécificité, qui nous semble mal retranscrite lorsqu'on utilise l'expression PME médiane, nous le conserverons tout au long de l'article. 


\begin{abstract}
The paper describes how the German Mittelstand, or small-and medium-sized entreprises, are financed in Germany. The first section of the paper documents the role of the German Mittelstand, both in a static and a dynamic framework, and contrasts it to that in other leading developed industrialized countries. In general, the Mittelstand has played a more important role in Germany than have small-and medium-sized enterprises in other developed industrialized nations such as the United States and the United Kingdom. The traditional success of the German Mittelstand is attributable to a system of finance which is richly layered by complementary institutions designed to meet the financial needs of both large and smaller enterprises. At the same time there is evidence that even under the German system of finance a negative relationship exists between liquidity constraints and firm size. And, it appears that the German system of finance is particularly vulnerable in its ability to channel funds into new firms in new industries.
\end{abstract}

\title{
RESUMEN
}

Este trabajo intenta describir cómo la Mittelstand, en otros terminos las pequeñas y medianas empresas, está financiada en Alemania. La primera parte expone el papel de la Mittelstand alemana desde un puntode vista estático y dynámico y establece las comparaciones con las situaciones observables en los principales paises industrials desarollados. De una forma general, nos damos cuenta de que la Mittelstand a jugado un papel más importante en Alemania que en paises como los Estados Unidos o Reino Unido. El éxito obtenido de la Mittelstand se puede ser explicado por referencia al sistema financiero alemán que se revela ámpliamente apoyado por instituciones adjuntas, instauradas para la satisfacción de las necessitades especificas de las grandes empresas y de las PyMEs. Paraélamente, es possible probas que a pesar de la existencia de un cierto sistema de financiamiento, existe una relacion entre el tamaño de las empresas y de la necessidad de líquido que éstas sufren. Parece finálmente que el sistema financiero alemán se revela particulámente con poco rendimiento en lo que concierne a la capacidad de destinar fondos a las empresas que nacen dentro de nuevos sectores de actividad.

\section{Introduction}

Une sorte de paradoxe concernant le système de financement de la Mittelstand allemande peut être observé. D'une part, il est légitime de considérer qu'en raison du développement d'un système d'institutions largement diversifié mettant en relation établissements financiers, autorités publiques et entreprises privées, le système de financement à l'œuvre en Allemagne constitue un modèle pour la distribution de fonds aux PME. Non seulement la Mittelstand se présente comme le pivot du miracle économique allemand et de la puissance économique qu'il a engendrée, mais, en outre, elle semble avoir joué un rôle 
encore plus important dans le développement économique allemand que dans celui d'autres économies telles que celles des États-Unis ou de la GrandeBretagne.

D'autre part, bien que la Mittelstand apparaisse comme la clé de voûte du succès économique germanique, un élément a systématiquement été omis au cours des dernières années; il s'agit de l'émergence de petites entreprises de haute technologie dans des secteurs naissants tels que l'informatique, les biotechnologies et la production de logiciels. Il est alors possible que l'insuffisante activité entrepreneuriale dans les secteurs de haute technologie soit directement causée par l'existence de rigidités et de contraintes dans l'offre de liquidité aux jeunes entreprises de ces nouveaux secteurs, ces dysfonctionnements étant inhérents à ce même système de financement.

En fait, on peut mettre en évidence deux aspects institutionnels du système financier allemand qui contrastent avec les pratiques américaines ou britanniques, ces deux facteurs étant susceptibles d'influencer les possibilités d'accès aux moyens de financement des PME. En premier lieu, les entreprises allemandes dépendent quasi exclusivement des banques en ce qui concerne l'attribution de ressources externes, le marché des capitaux externes demeurant quelque peu sous-développé. En second lieu, non seulement les banques représentent les principaux intermédiaires financiers capables de procurer des ressources externes aux entreprises, mais elles sont également fortement représentées dans les conseils d'administration des sociétés. Cette spécificité du système financier allemand qui intègre finance et contrôle est repérée par Cable (1985, p. 119) à l'aide de l'expression «marché quasi interne du capital ».

Ce travail a pour objectif de procéder à une analyse du système de financement allemand et de mettre l'accent sur les modalités de financement de la Mittelstand. De plus, nous évaluerons la robustesse des éléments fondant l'appréciation d'efficience portée à l'égard du système de financement de la Mittelstand allemande.

Dans la deuxième partie, nous analyserons le rôle des PME en Allemagne, tant d'un point de vue statique que dynamique. Nous montrerons que la Mittelstand joue un rôle plus important en Allemagne que ne le jouent les PME au Royaume-Uni et aux États-Unis. Dans la troisième partie sera examiné le système financier. La mise en évidence des conséquences de ce dernier sur le financement des PME est au centre de notre quatrième section, alors que la cinquième soulève le problème de l'efficience du système de financement des PME en Allemagne. Le résumé et la conclusion qui composent la sixième section nous conduisent à considérer que la force du système de financement allemand réside dans l'affectation de ressources aux entreprises bien implantées appartenant à des secteurs traditionnels, qu'elles soient de petite ou grande 
dimension, alors qu'il se révèle moins performant lorsqu'il s'agit de financer les entreprises en création appartenant à des secteurs de haute technologie.

\section{Le rôle de la Mittelstand dans l'économie allemande}

\section{1. Évaluation}

L'une des appréciations les plus couramment portées sur l'Allemagne est la domination exercée par un petit nombre de très grandes sociétés telles que Daimler-Benz, Siemens et Wolkswagen. Alors que ces sociétés sont effectivement très grandes et dominantes, y compris à l'aune des standards internationaux, l'économie allemande est également plus riche et plus complexe qu'on pourrait le croire au premier abord.

Quel est donc précisément le rôle des PME ou de ce qui est désormais plus connu sous le nom de Mittelstand ? Comme dans de nombreux autres pays, l'évaluation de la contribution des PME se révèle une tâche à la fois délicate et importante, car le rôle effectif de la Mittelstand ne peut pas être précisément apprécié sans porter une attention particulière aux problèmes de mesure et de quantification.

\subsection{Le rôle en statique}

L'Institut de statistiques sur l'industrie (Arbeitsstättenzähung) fournit les sources les plus sûres et les plus complètes pour mesurer les niveaux d'emploi en fonction de la taille des établissements. La collecte de telles données commencée en 1882 a été élargie, dès 1907, de manière à intégrer les données d'entreprises et d'établissement, le mode d'organisation le plus récent de l'Institut datant de 1987.

Selon l'Institut de statistiques, ce que l'on a coutume de considérer comme faisant partie de la classe des PME, définie comme l'ensemble des entreprises employant moins de 100 salariés, représente plus de la moitié de l'emploi du secteur privé non agricole. En outre, plus d'un tiers de l'ensemble de l'effectif salarié travaille dans des entreprises employant moins de 20 personnes alors qu'à peine plus du quart de la main-d'œuvre travaille dans des firmes comptant plus de 100 salariés (Fritsch, 1993).

Le tableau 1 procède à la distinction de l'effectif employé au niveau de l'entreprise et de l'établissement. Ce qui n'est pas pour étonner, la proportion d'emplois dans les établissement de type PME dépasse celle des firmes de moyenne dimension. 


\section{TABLEAU 1}

Distribution des emplois selon la taille de l'entreprise et celle de l'établissement, 1987, en pourcentages*

\begin{tabular}{|c|c|c|c|c|c|c|}
\hline \multirow{2}{*}{$\begin{array}{c}\begin{array}{c}\text { Classe } \\
\text { de taille }\end{array} \\
\begin{array}{c}\text { Nombre } \\
\text { d'employés }\end{array}\end{array}$} & \multicolumn{2}{|c|}{$\begin{array}{c}\text { Tous secteurs } \\
\text { confondus }\end{array}$} & \multicolumn{2}{|c|}{ Industrie } & \multicolumn{2}{|c|}{ Services } \\
\hline & Établissements & Entreprises & Établissements & Entreprises & Établissements & Entreprises \\
\hline $1-4$ & 15,0 & 13,3 & 5,7 & 5,3 & 23,4 & 21,1 \\
\hline $5-9$ & $\begin{array}{c}13,1 \\
(28,1)\end{array}$ & $\begin{array}{c}11,6 \\
(24,9)\end{array}$ & $\begin{array}{c}8,2 \\
(13,9)\end{array}$ & $\begin{array}{c}7,6 \\
(12,8)\end{array}$ & $\begin{array}{c}17,6 \\
(41,0)\end{array}$ & $\begin{array}{c}15,5 \\
(36,6)\end{array}$ \\
\hline $10-19$ & $\begin{array}{c}10,9 \\
(39,0)\end{array}$ & $\begin{array}{c}9,4 \\
(34,4)\end{array}$ & $\begin{array}{c}9,4 \\
(23,3)\end{array}$ & $\begin{array}{c}8,8 \\
(21,6)\end{array}$ & $\begin{array}{c}12,1 \\
(53,1)\end{array}$ & $\begin{array}{c}9,9 \\
(46,5)\end{array}$ \\
\hline $20-49$ & $\begin{array}{c}11,7 \\
(50,7)\end{array}$ & $\begin{array}{c}9,5 \\
(43,8)\end{array}$ & $\begin{array}{c}11,5 \\
(34,3)\end{array}$ & $\begin{array}{c}10,2 \\
(31,8)\end{array}$ & $\begin{array}{c}11,8 \\
(65,0)\end{array}$ & $\begin{array}{c}8,8 \\
(55,3)\end{array}$ \\
\hline $50-99$ & $\begin{array}{c}8,4 \\
(59,1)\end{array}$ & $\begin{array}{c}6,6 \\
(57,0)\end{array}$ & $\begin{array}{c}9,1 \\
(43,4)\end{array}$ & $\begin{array}{c}7,6 \\
(39,4)\end{array}$ & $\begin{array}{c}7,9 \\
(80,4)\end{array}$ & $\begin{array}{c}5,5 \\
(66,0)\end{array}$ \\
\hline $100-199$ & $\begin{array}{c}8,6 \\
(67,7)\end{array}$ & $\begin{array}{r}6,6 \\
(57,0)\end{array}$ & $\begin{array}{c}9,9 \\
(53,3)\end{array}$ & $\begin{array}{c}8,0 \\
(47,3)\end{array}$ & $\begin{array}{c}7,5 \\
(80,4)\end{array}$ & $\begin{array}{c}5,3 \\
(66,0)\end{array}$ \\
\hline $200-499$ & $\begin{array}{c}11,2 \\
(78,9)\end{array}$ & $\begin{array}{c}8,8 \\
(65,8)\end{array}$ & $\begin{array}{c}13,6 \\
(66,9)\end{array}$ & $\begin{array}{c}10,6 \\
(57,9)\end{array}$ & $\begin{array}{c}9,1 \\
(89,4)\end{array}$ & $\begin{array}{c}7,0 \\
(73,09)\end{array}$ \\
\hline $500-999$ & $\begin{array}{c}7,0 \\
(85,9)\end{array}$ & $\begin{array}{c}5,9 \\
(71,6)\end{array}$ & $\begin{array}{c}8,9 \\
(75,8)\end{array}$ & $\begin{array}{c}7,1 \\
(65,0)\end{array}$ & $\begin{array}{c}5,3 \\
(94,7)\end{array}$ & $\begin{array}{c}4,7 \\
(77,7)\end{array}$ \\
\hline + de 1000 & $\begin{array}{c}14,1 \\
(100)\end{array}$ & $\begin{array}{c}28,5 \\
(100)\end{array}$ & $\begin{array}{c}23,8 \\
(100)\end{array}$ & $\begin{array}{c}35,0 \\
(100)\end{array}$ & $\begin{array}{c}5,3 \\
(100)\end{array}$ & $\begin{array}{c}22,3 \\
(100)\end{array}$ \\
\hline
\end{tabular}

Source: Adapté de Fritsch (1993)

* Valeurs cumulées entre parenthèses.

Seul est comptabilisé l'emploi du secteur privé non agricole.

Une série d'études réalisées par Schwalbach (1989), Fritsch (1993), Stockman et Leicht (1994) procèdent à la comparaison de différentes mesures de l'activité économique en isolant le rôle exact des $\mathrm{PME}^{2}$. Ces études rendent en général compte d'une distribution de l'activité économique par classe d'entreprises similaires, que l'emploi ou le montant des ventes soit utilisé comme critère.

Comment comparer la part de l'activité attribuable à la Mittelstand aux résultats obtenus dans d'autres pays industrialisés développés? Acs et Audretsch (1993, tableau 12.1) utilisent comme critère de référence l'emploi industriel des entreprises de moins de 500 salariés de manière à comparer le rôle

2. Les recherches de Bannasch (1990 et 1993) portent également sur cette question en intégrant l'ex-Allemagne de l'Est. 
respectivement joué par les PME dans un grand échantillon de pays industrialisés. Ils montrent qu'aux États-Unis 35,2\% de l'emploi industriel recensé en 1987 est le fait des PME. Pour l'année 1986, la part de l'emploi créé par ces entreprises s'élève à $39,9 \%$ au Royaume-Uni (1986) et à $71,8 \%$ au Portugal. Comme on peut le voir à partir du tableau $1,57,9 \%$ de l'emploi industriel allemand se trouve dans des entreprises comptant moins de 500 salariés, ce qui permet de considérer que la part de l'activité économique industrielle reliée à la Mittelstand est considérablement plus élevée en Allemagne qu'aux ÉtatsUnis ou au Royaume-Uni, mais plus faible qu'au Portugal, qu'en Italie ou qu'au Japon ${ }^{3}$. Au total, il ne fait donc aucun doute que la Mittelstand allemande représente une part importante de l'activité économique.

\subsection{Une perspective dynamique}

La Mittelstand a-t-elle vu son importance croître ou diminuer dans le temps? Acs et Audretsch (1993) montrent que, probablement comme dans tous les principaux pays industrialisés, la part de l'emploi industriel dans les PME a augmenté entre le début des années 1970 et la fin de la décennie 1980. Plus précisément, la Mittelstand représentait 57,9\% des emplois dans l'industrie en 1987 contre $54,8 \%$ en 1970 . Cette progression ne se répartit pas de manière équiproportionnelle entre les secteurs; comme le montre le schéma 1, alors que la variation de l'activité économique entre 1970 et 1987 favorisait les PMI au détriment des GE, le secteur des services s'est caractérisé par un mouvement inverse (Fritsch, 1993).

\subsection{Le rôle qualitatif}

Simon (1990) a étudié ce qu'il a appelé les «champions allemands cachés», à savoir les entreprises de la Mittelstand réalisant les meilleures performances à l'exportation. Ces sociétés possèdent une part de marché de $22,6 \%$ pour le produit considéré, ce chiffre passant à $31,7 \%$ lorsqu'on se limite à l'Europe. Ces compagnies possèdent en moyenne 9,6 filiales étrangères, ce qui apparaît comme un chiffre extrêmement élevé eu égard à la taille relativement modeste des sociétés mères.

Parallèlement mérite d'être mentionné l'absence notable de créations d'entreprises dans les secteurs de haute technologie et des nouvelles technologies de l'information et de la communication. La force de la Mittelstand allemande reflète ainsi particulièrement bien la puissance de l'économie allemande dans les secteurs industriels traditionnels.

3. Pour plus de précision, $c f$. Acs et Audretsch (1993) et Loveman et Sengenberger (1991). 


\section{SCHÉMA 1}

Variation dans la structure par taille des entreprises des secteurs secondaire et tertiaire sur la période 1970-1987

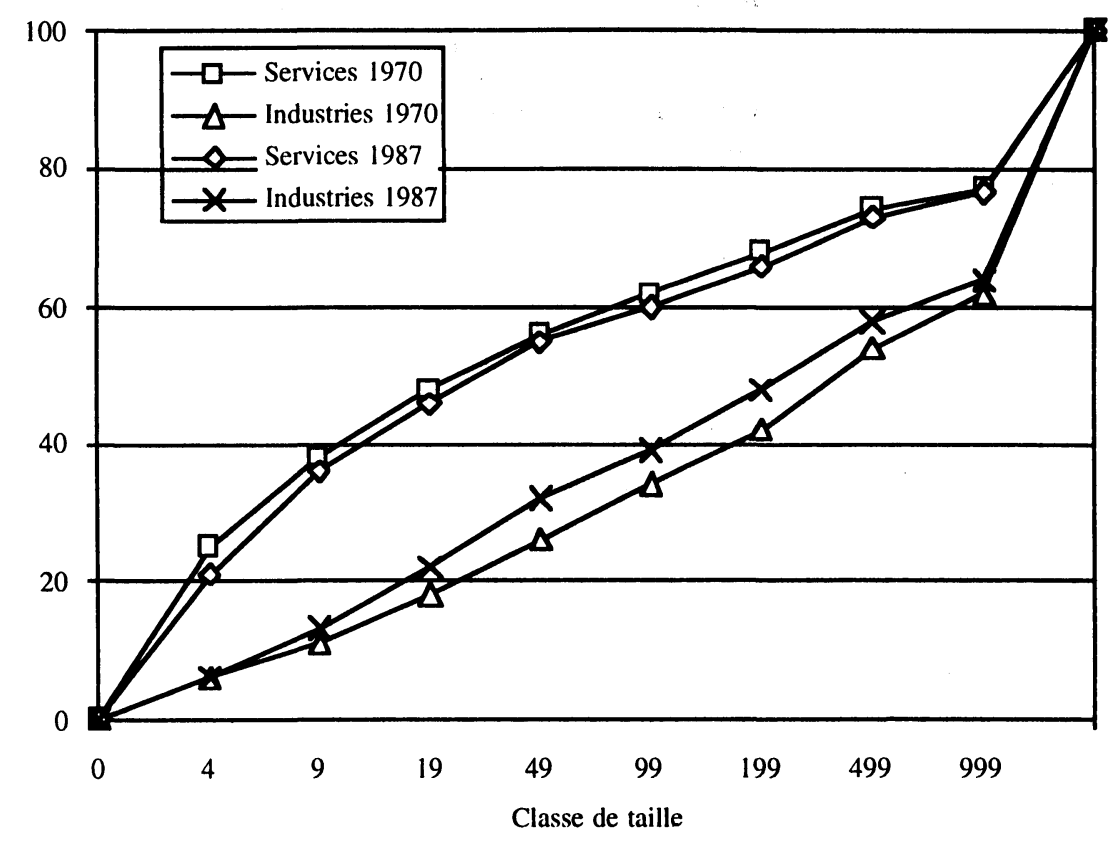

\section{Le système financier allemand}

\subsection{Les sources de financement}

Compte tenu de l'opinion généralement admise sur l'importance des prises de participation des banques dans l'industrie allemande, il est surprenant de constater ( $c f$. le tableau 2) que l'essentiel des financements est d'origine interne et non externe; cette tendance se révélant particulièrement marquée lorsqu'on observe les 100 plus grandes entreprises. La finance interne semble donc jouer un rôle nettement plus important qu'aux États-Unis, au Japon ou au Royaume-Uni.

Au cours des années 1980, environ $90 \%$ du financement de la formation brute de capital physique ou financier comptabilisée pour les grandes entreprises cotées en Bourse a été réalisé grâce à des ressources internes. Comme élément de comparaison, citons le cas du Royaume-Uni où seulement $58 \%$ du montant de ces opérations relève d'un financement interne. En termes de sources de financement affectées au seul capital physique, les fonds d'origine interne s'élèvent à $138 \%$ pour les entreprises allemandes alors qu'ils ne représentent que $113 \%$ pour les entreprises britanniques. 


\section{TABLEAU 2 \\ Montants bruts et nets des ressources financières affectées à l'investissement par les entreprises américaines, japonaises, allemandes et anglaises sur la période 1970-1989 (en pourcentage)*}

\begin{tabular}{|c|c|c|c|c|}
\hline & États-Unis & Japon & Allemagne & Grande-Bretagne \\
\hline \multicolumn{5}{|l|}{ Montants bruts } \\
\hline $\begin{array}{l}\text { Autofinancement } \\
\text { Nouveaux emprunts } \\
\text { Nouvelles actions } \\
\text { Autres }\end{array}$ & $\begin{array}{r}66,9 \\
41,2 \\
0,8 \\
-8,8\end{array}$ & $\begin{array}{r}33,7 \\
62,1 \\
3,5 \\
0,7\end{array}$ & $\begin{array}{r}55,2 \\
24,0 \\
2,1 \\
18,6\end{array}$ & $\begin{array}{r}72,0 \\
25,0 \\
4,9 \\
-2,0\end{array}$ \\
\hline \multicolumn{5}{|l|}{ Montants nets } \\
\hline $\begin{array}{l}\text { Autofinancement } \\
\text { Nouveaux emprunts } \\
\text { Nouvelles actions } \\
\text { Autres }\end{array}$ & $\begin{array}{r}85,9 \\
34,6 \\
1,1 \\
-21,5\end{array}$ & $\begin{array}{r}57,9 \\
41,3 \\
4,6 \\
-3,8\end{array}$ & $\begin{array}{r}70,9 \\
9,0 \\
0,6 \\
19,4\end{array}$ & $\begin{array}{r}102,4 \\
5,4 \\
-3,3 \\
-4,4\end{array}$ \\
\hline $\begin{array}{l}\text { Montants bruts } \\
\text { (100 plus grandes } \\
\text { entreprises)** }\end{array}$ & $1982-1987$ & N.D. & $1982-1988$ & $1982-1988$ \\
\hline $\begin{array}{l}\text { Autofinancement } \\
\text { Nouveaux emprunts } \\
\text { Nouvelles actions } \\
\text { Autres }\end{array}$ & $\begin{array}{r}51,5 \\
30,2 \\
10,1 \\
8,1\end{array}$ & & $\begin{array}{r}89,6 \\
2,2 \\
8,2 \\
\text { N.D. }\end{array}$ & $\begin{array}{l}58,2 \\
27,5 \\
14,3 \\
\text { N.D. }\end{array}$ \\
\hline \multicolumn{5}{|l|}{$\begin{array}{l}\text { Montants nets } \\
\text { ( } 100 \text { plus grandes } \\
\text { entreprises)** }\end{array}$} \\
\hline $\begin{array}{l}\text { Autofinancement } \\
\text { Nouveaux emprunts } \\
\text { Nouvelles actions } \\
\text { Autres }\end{array}$ & $\begin{array}{r}79,1 \\
3,2 \\
3,1 \\
14,6\end{array}$ & $\begin{array}{c}50,9 \\
19,0 \\
30,0 \\
\text { N.D. }\end{array}$ & $\begin{array}{l}137,9 \\
-27,8 \\
-10,2 \\
\text { N.D. }\end{array}$ & $\begin{array}{r}112,9 \\
-1,6 \\
-11,3 \\
\text { N.D. }\end{array}$ \\
\hline
\end{tabular}

Sources : Statistiques financières de l'OCDE.

N.D. : Données non disponibles

Colin, Mayer et Ian Alexander (1990), «Banks and securities markets : corporate financing in Germany and the United Kingdom », Journal of Japanese and International Economics, vol. 4.

Bronwyn, H. Hall, (1994), «Corporate restructuring and investment horizons in the United States, 1976-1987 », Business History Review, printemps, nº 68.

* Les définitions des variables peuvent varier d'un pays à l'autre en fonction des pratiques comptables.

** Les données américaines et japonaises portent sur les 100 plus grandes entreprises. En ce qui concerne les statistiques allemandes et britanniques, sont prises en compte les plus grandes sociétés non financières parmi l'ensemble des 115 plus grandes firmes du pays considéré. 
Bien que la finance interne constitue le mode dominant de réalisation de l'investissement pour l'ensemble du pays, l'endettement, qui représente en moyenne $24 \%$ des fonds dont disposaient les unités productives sur la période 1970-1989, constitue le mode de financement externe privilégié par la plupart des entreprises. Sur les 100 plus grandes sociétés, qui sont précisément celles possédant les meilleures possibilités d'accès aux marchés d'actifs financiers nationaux et internationaux, et sur le même intervalle de temps, seulement $2,2 \%$ avaient recours à l'endettement comme mode de financement. Ce résultat est donc cohérent avec l'idée selon laquelle les firmes allemandes substituent l'autofinancement ou le financement par actions à l'endettement dès qu'elles en ont la possibilité.

En Allemagne, le marché pour le contrôle des entreprises ne peut être, au mieux, que limité. Comme l'ont démontré Mayer et Alexander (1990), le ratio de paiement de dividendes, défini comme les dividendes bruts rapportés aux profits non accompagnés de distribution, s'élevait à $13 \%$ pour les grandes entreprises allemandes, mais atteignait $31 \%$ pour leurs homologues britanniques au cours des années $1980^{4}$. Le rôle mineur, voire négligeable, joué par le marché en matière de contrôle d'entreprises est confirmé par le nombre relativement faible de sociétés de droit public. En 1989, seulement 2682 S.A., appelées Aktiengesellschaften ou AGs, étaient dénombrées en Allemagne et, parmi ces sociétés, 501 faisaient l'objet d'une cotation (Banque Fédérale d'Allemagne, 1991b). Cependant, comme l'indiquent Edwards et Fischer (1994), l'essentiel des plus grandes compagnies allemandes sont des Aktiengesellschaften, puisque sur les 100 plus grandes entreprises, 88 sont des S.A.

Les entreprises financières et non financières détiennent et influencent largement les entreprises allemandes. En 1988, les entreprises non financières représentaient $39,9 \%$ de la valeur de capitalisation nominale totale des S.A., les banques contribuant pour 11,6\% (Banque Fédérale d'Allemagne, 1991b). Edwards et Gordon (1994) observent à ce propos qu'un ensemble de modalités, incluant les holdings et les votes à la place des proches, le droit des sociétés, la loi sur la codétermination, la réglementation boursière et le système de régulation financière, a évolué de manière à favoriser les prises de contrôle des sociétés en Allemagne où les plus grandes entreprises industrielles occupent une position clé.

4. Mayer et Alexander (1990) soulignent que même si le nombre de fusions de grande envergure réalisées en Allemagne est élevé, ces opérations sont généralement négociées. À ce jour, aucune offre publique d'achat hostile n'a été réussie. 


\subsection{Le contrôle par les banques}

Le système de vote à la place des proches, ou Depotstimmrecht, autorise les banques à voter sur les parts d'entreprises détenues par leurs clients. En plaçant les actions qu'ils détiennent sur le compte d'un administrateur, le client transfère en effet son droit de vote à la banque. Non seulement environ la moitié des actions assorties d'un droit de vote sont déposées sur de tels comptes mais, en outre, le contrôle sur les actions avec droit de vote exercé par les plus grands établissements bancaires a eu d'importantes conséquences sur les S.A. possédant un large actionnariat (Edwards et Fischer, 1994).

La revue The Economist signale que $85 \%$ des 171 plus grandes entreprises industrielles et commerciales allemandes cotées possèdent un actionnaire unique détenant plus de $25 \%$ des voix ; mais c'est seulement dans $6 \%$ des cas que cet actionnaire est une banque.

Il n'est pas davantage évident que les entreprises allemandes soient aussi fortement dépendantes des banques pour la satisfaction de leurs besoins de financement, comme on le croit fréquemment. Par exemple, Edwards et Fischer (1994) montrent qu'entre 1950 et 1989 les emprunts auprès des banques n'ont contribué qu'à la réalisation de $12 \%$ des investissements en actifs physiques effectués par les firmes du secteur industriel.

Toutefois, les études d'Edwards et Fischer, d'une part, et de Franks et Mayer, d'autre part, peuvent sous-estimer l'importance de l'influence exercée par les banques. Ce biais s'explique par le fait que les banques exercent leur droit de vote à la place des proches dans les entreprises pour le compte d'une partie de leurs clients et en leur nom, alors qu'elles possèdent leurs propres armes de gestion des financements, ce qui leur permet d'exercer un contrôle sur les entreprises sans avoir besoin de détenir elles-mêmes d'importants stocks d'actions. Plus précisément, les sociétés possédant un large actionnariat dans lequel aucune position dominante n'apparaît sont particulièrement vulnérables au contrôle par une banque. Lorsque ce contrôle indirect est pris en considération, on s'aperçoit que le contrôle qu'exercent les banques sur les principales sociétés allemandes est considérable. Ainsi, en 1992, les banques contrôlaient les droits de vote de grandes sociétés dans les proportions suivantes:

$-95,5 \%$ de Siemens ;

$-44,1 \%$ de Volkswagen;

$-98,5 \%$ de Hoechst;

$-94,7 \%$ de BASF ;

$-91,3 \%$ de Bayer;

$-45,4 \%$ de Thyssen et $98,1 \%$ de Mannesmann 5 .

5. «Those German banks and their industrial treasures », The Economist, 21 janvier 1995, p. 77-78. 
Suivant le droit allemand des sociétés, les S.A. doivent être dirigées par un comité de gestion (Vorstand) et un conseil de surveillance (Aufsichtsart) ${ }^{6}$ et, dans le cas où l'entreprise emploie plus de 2000 salariés, la législation sur la cogestion (Mitbestimmung) impose que la moitié des membres du conseil de surveillance soient des représentants des salariés?

Enfin, les trois grandes banques répertoriées (la Deutsche Bank, la Dresdner Bank et la Commerzbank) votent de manière systématique en faveur du groupe des dirigeants en place. Toujours selon Edwards et Fischer (1994), cette relation harmonieuse entre les trois grandes banques et les plus grandes entreprises n'est que le reflet de la tendance croissante de la congruence et du rapprochement des intérêts des banques et des entreprises industrielles.

\subsection{La structure du passif}

Le système de financement allemand se caractérise par un recours exceptionnellement élevé à la dette. Les ratios endettement/fonds propres y sont de l'ordre de 4 pour 1 alors que le rapport n'est que de 1,3 à 1 dans le cas des sociétés américaines ${ }^{8}$. Le tableau 3 rend compte de l'augmentation du recours à la dette des entreprises allemandes sur la période 1971-1989.

TABLEAU 3

Structure du passif des entreprises allemandes, 1971-1989 (en pourcentage du total)

\begin{tabular}{lccc}
\hline & 1971 & 1981 & 1989 \\
\hline Capitaux propres & 26 & 19 & 19 \\
Dettes & 74 & 81 & 81 \\
dont & 42 & 48 & 44 \\
- à court terme & 22 & 19 & 16 \\
- à long terme & 10 & 14 & 21 \\
- provisions* & & & \\
\hline
\end{tabular}

Sources : Les chiffres pour les années 1971 et 1981 sont extraits de «Business Finance in the UK and Germany ", Deutsche Bundesbank, 1984, vol. 36, n⿳ 11, p. 3. Ceux de l'année 1989 proviennent de "West German enterprises' profitability in 1989 », Monthly Report of the Deutsche Bundesbank, 1990, vol. 42, nº 11, p. 21. Adapté de Deeg (1993).

* Réserves pour dettes futures.

6. La législation interdit à une même personne d'être simultanément membre de ces deux conseils.

7. Cette même législation exige que dans les compagnies comptant moins de 2000 salariés, au moins un tiers des sièges du conseil de surveillance soient occupés par des représentants des salariés.

8. "Suddenly, Germans love to hate their banks », Business Week, 20 février 1995, p. 42. Voir aussi "Business finance in UK and Germany», Bundesbank Monthly Reports, novembre 1994, p. 36. 
De manière générale, on constate que sur la longue période la structure de la demande de financements externes a évolué, le recours des plus grandes entreprises à la finance interne ayant significativement augmenté depuis le début des années 1970. De l'examen des sources et de l'utilisation des fonds des entreprises industrielles entre 1970 et 1989, Edwards et Fischer (1994) déduisent que la rétention des bénéfices a permis de financer $62 \%$ de l'investissement effectué par les entreprises. En revanche, les crédits bancaires n'ont contribué qu'à la réalisation de $18 \%$ de cet investissement. En fait, si l'on ne prend en considération que l'acquisition d'actifs corporels, on constate qu'à peine $11 \%$ des financements proviennent de banques allemandes 9 .

\section{Le financement des PME}

\subsection{Les institutions financières}

Bien que le rôle joué par les grandes banques dans le financement des grandes sociétés par actions industrielles ait suscité un fort intérêt ${ }^{10}$, une attention nettement moins grande a été accordée aux autres institutions constitutives du système financier allemand. Vitols (1994) souligne ainsi qu'en réalité, les trois grandes banques allemandes ne représentent que moins d'un dixième de l'actif bancaire total ${ }^{11}$. L'essentiel des crédits distribués par les trois grandes (banques privées) est affecté aux plus grandes entreprises. Selon Vitols (1994, p. 7) «ces banques ont traditionnellement limité leur activité de crédits à l'industrie aux plus grandes sociétés par actions».

Les institutions financières les plus importantes sont les Sparkassen, ou caisses d'épargne, dont l'activité principale consiste dans la collecte de dépôts auprès du public, et les Genossenschaftsbanken, des établissements de nature mutualiste. Bien que les Sparkassen représentent environ $40 \%$ du total des actifs bancaires, les Genossenschaftsbanken y contribuent pour approximativement $15 \%$ (Deeg, 1992). L'activité de ces institutions financières est essentiellement orientée vers le financement de la Mittelstand. Vitols (1994) remarque que la majeure partie des fonds affectés aux entreprises de moyenne dimension proviennent des Sparkassen, qui représentent un tiers du système des banques de dépôt, lequel est constitué de trois grands sous-ensembles:

9. Pour comparaison, on peut signaler que les firmes britanniques financent $24 \%$ de leurs investissements grâce à des crédits bancaires (Edwards et Fischer, 1994).

10. Voir, par exemple, Cable (1985).

11. Le rapport mensuel de la Deutsche Bundesbank (avril 1989, p. 15, tableau 4.1) montre que la part de marché des trois grandes est passée de $10,2 \%$ en 1970 à $10,6 \%$ en 1978 pour finalement atteindre $8,9 \%$ en 1988 . 
1. Le premier tiers inclut environ 750 banques de dépôt publiques. Ces caisses d'épargne sont la plupart du temps détenues par la municipalité ou le département dans lequel elles sont implantées. Les autorités publiques locales et les caisses d'épargne entretiennent ainsi des relations de dépendance réciproque, les principaux objets d'affectation des crédits qu'elle distribuent ayant pour origine les entreprises de la région, si bien que ces institutions œuvrent en faveur d'un développement local. Vitols (1994) observe à ce propos que les caisses d'épargne financent abondamment les parcs scientifiques ainsi que d'autres projets relevant du développement local.

2. Le deuxième tiers se compose d'intermédiaires financiers parmi lesquels on peut citer les Landesbanken, ou banques régionales, propriétés des États et des associations régionales de banques de dépôt. Ces banques régionales permettent aux agents non financiers d'avoir accès aux marchés de capitaux nationaux et internationaux, grâce à l'existence de services spécialisés chargés de prodiguer des conseils de gestion et d'assurer les opérations de courtage au profit des Sparkassen. En raison de leur taille relativement restreinte, la plupart des banques de dépôt d'envergure locale n'ont pas accès à l'intégralité des segments composant les marchés d'actifs domestiques ou étrangers. En outre, leur capacité de proposer et de développer des services spécialisés se révèle limitée. Le rôle de la structure institutionnelle que constitue ce deuxième tiers est, par conséquent, essentiellement destiné à combler le vide existant entre les petites banques locales et les marchés.

3. Le troisième tiers se compose d'institutions intervenant à l'échelle nationale. Ces établissements permettent aux agents non financiers de se présenter sur les marchés du crédit tant nationaux qu'internationaux.

Les groupes de banques mutualistes sont longtemps demeurés spécialisés dans le financement de l'agriculture et de l'artisanat et ont largement contribué au développement industriel observé au cours du XIX ${ }^{\mathrm{e}}$ siècle. À l'instar des banques de dépôt, le secteur bancaire mutualiste peut être scindé en trois niveaux.

1. Le niveau inférieur comprend 3400 banques dont la plupart sont détenues et gérées par des PME.

2. Le niveau intermédiaire, ou régional, procure au troisième tiers des services financiers spécialisés surtout dans le refinancement et offre des programmes de formation.

3. Le niveau supérieur, c'est-à-dire national, offre des services semblables à ceux que proposent les établissement inclus dans le tiers précédent, 
mais constituent en outre une structure d'associations de banques mutualistes (Deeg, 1992).

Comme on peut le voir au tableau 4, la part de crédits consentis aux entreprises par les trois grandes a fortement diminué, passant de $15,4 \%$ en 1972 à $11,9 \%$ en 1982. Au cours de la même période, les banques de dépôt ont vu leur part de marché passer de 33,2 à 36,7\%, alors que celle des groupes bancaires mutualistes qui s'élevait à $12,9 \%$ en 1972 montait à $17,4 \%$ en 1982 .

\section{TABLEAU 4}

Crédits aux entreprises distribués par les principaux groupes bancaires (1972-1990)

\begin{tabular}{lcccc}
\hline Types de banques & Année & $\begin{array}{c}\text { Crédits } \\
\text { à toutes } \\
\text { les entreprises* }\end{array}$ & $\begin{array}{c}\text { Crédits } \\
\text { aux firmes } \\
\text { industrielles }\end{array}$ & $\begin{array}{c}\text { Crédits } \\
\text { aux PME }\end{array}$ \\
\hline Commerciales & 1972 & 36,7 & 54,2 & \\
& 1982 & 30,1 & 38,8 & \\
\hline (Les trois grandes) & 1990 & 31,3 & 42,1 & 28,3 \\
& 1972 & 15,4 & 28,2 & 37,2 \\
& 1982 & 11,9 & 18,2 & 36,7 \\
\hline Caisses d'épargne & 1990 & 13,8 & 22,4 & 28,4 \\
& 1972 & 33,2 & 26,1 & \\
\hline Coopératives & 1982 & 36,7 & 33,2 & 61,8 \\
& 1990 & 33,6 & 30,6 & \\
\hline
\end{tabular}

Sources: Les chiffres pour les années 1972 et 1972 sont tirés de K.J. Lubitz (1984), Bankmarketing gegenüber mittelständischen Betrieben, Francfort, Fritz Knapp, p. 11. Les données pour l'année 1990, de la Deutsche Bundesbank, 1991. Adapté de Deeg (1993).

Notes: Les pourcentages peuvent avoir un total inférieur à 100 , car tous les crédits bancaires ne sont pas recensés.

* Les chiffres incluent les crédits hypothécaires consentis et intègrent toutes les entreprises artisanales.

** Ces pourcentages sont calculés en excluant les crédits hypothécaires, ils ne concernent donc que les sociétés du secteur industriel.

*** Les chiffres mentionnés représentent le pourcentage de crédits affectés aux PME parmi l'ensemble des crédits distribués aux entreprises. Les crédits aux PME comprennent les crédits hypothécaires d'un montant inférieur à un million de DM et les crédits requérant un engagement de la banque inférieur à un million de DM.

À partir du milieu des années 1970, les banques de dépôt sont devenues, à la place du secteur bancaire privé, les principales institutions distributrices 
de crédits aux entreprises. Quoique le pouvoir économique et politique des trois grandes, particulièrement en termes d'offre de moyens de financement et de participation à la gestion des grandes entreprises nationales, ait capté l'attention des observateurs étrangers, l'importance quantitative et le rôle clé joué par les autres institutions bancaires dans le paysage financier allemand, notamment en ce qui concerne l'offre de fonds aux PME, se présentent comme un facteur explicatif déterminant du développement économique allemand.

Le plus important changement pouvant être observé dans le temps relève de l'augmentation de la part de crédits distribués par les trois grandes à la Mittelstand. En effet, en 1972, 28,3\% des crédits consentis par les grandes banques allemandes étaient affectés à des PME, alors qu'en 1982 la proportion s'élevait à $37,2 \%$. Parallèlement, la part de crédits offerts par les banques de dépôt et les réseaux mutualistes connaissait une diminution. Ainsi, alors que $36,7 \%$ des prêts distribués par les caisses d'épargne étaient destinés à la Mittelstand en 1972, dix ans plus tard, les PME ne recevaient plus que $28,4 \%$ des crédits offerts par ces établissements. De manière similaire, alors que les banques mutualistes affectaient l'essentiel des fonds prêtables offerts, soit $91,8 \%$, aux entreprises de taille moyenne en 1972, la proportion de crédits consentis par ces réseaux à la Mittelstand avait diminué d'un tiers, passant à $67,4 \%$ en 1982.

Cabel, Griffin et Deeg (1993) remarquent que le développement de la distribution de crédits à la Mittelstand par les grandes banques résulte d'un changement des stratégies bancaires délibérément instauré. Selon ces auteurs,

[...] la stratégie initiale des grandes banques consistait à asseoir leur développement sur le fer de lance de l'économie allemande, c'est-à-dire les entreprises de dimension petite et moyenne repérées sous le terme générique de Mittelstand. Les grandes banques ont longtemps financé les exportations de cet ensemble de firmes. Toutefois, les réseaux de banques de dépôt et le secteur mutualiste les dépassaient de beaucoup en ce qui concerne le financement de l'activité industrielle à vocation domestique. L'acquisition d'une autonomie de décision croissante par les entreprises de type sociétés anonymes a alors suscité l'intérêt des banques commerciales à l'égard de la Mittelstand qui devenait une clientèle potentielle attractive. La Mittelstand dépendait fortement de l'offre de crédit. Dans le nouvel environnement financier caractérisé par le développement de marchés instables, ces firmes pouvaient espérer bénéficier d'un avantage informationnel une fois obtenu l'actionnariat que constitue la clientèle des banques. (Cabel, Griffin et Deeg, 1993, p. 17)

L'existence de ces intermédiaires financiers en ce qui concerne des montants élevés de ressources à la Mittelstand a contribué à favoriser l'accès des petites banques à des modes de financement à long terme et à taux fixe. Ces mécanismes intègrent les éléments suivants: 
1. des établissements de crédits spécialisés émetteurs d'obligations sur les marchés nationaux, ces titres permettant de refinancer les crédits longs à taux fixe consentis aux PME;

2. des possibilités de refinancement et de partage des risques réunissant les caisses de Dépôt et les banques mutualistes ;

3. des mécanismes facilitant le passage d'une large partie de l'épargne à long terme effectuée auprès des compagnies d'assurance vers les établissements bancaires par le biais des obligations émises par ces dernières.

Deux tiers environ des crédits longs distribués par les banques aux PME sont refinancés au moyen des trois mécanismes précédemment mentionnés (Vitols, 1994).

\subsection{Les financements publics}

De nombreuses mesures de politiques économiques ont vocation d'alimenter la Mittelstand en ressources financières. Ainsi, le Kreitanstalt für Wiederhaufbau, créé à la fin de la Seconde Guerre mondiale dans le but de faciliter la reconstruction, a vu ses orientations modifiées de manière à contribuer au développement technologique des entreprises de petite et moyenne dimension (Ministère fédéral de la Recherche et de la Technologie, 1985). Les entreprises dont le chiffre d'affaires annuel ne dépasse pas 590 millions de dollars ont la possibilité d'être subventionnées jusqu'à concurrence de $40 \%$ du coût des investissements consacrés au développement ou à la création technologique. De plus, les PME ne sont pas concernées par la plupart des textes constitutifs des lois antitrust allemandes (Gesetz Gegen Wettbewerbsbeschränkungen) de manière à faciliter la coopération dans le cadre d'opérations de marketing, d'achat et de R-D (Ministère de l'Économie, 1987; Audretsch, 1989).

Plus récemment encore, dans le but de contribuer au financement des PME implantées dans les cinq nouveaux États anciennement constitutifs de la République démocratique allemande, les autorités régionales et nationales ont mobilisé 55 milliards de dollars sous la forme de crédits directement affectés aux projets d'investissement, de subventions et aides ainsi que de réduction d'impôts accordés aux créateurs d'entreprises ${ }^{12}$. Le principal vecteur de distribution de ces moyens financiers est un fonds de crédit initialement créé grâce au milliard et demi de dollars que l'Allemagne a reçu des États-Unis dans le cadre du plan Marshall autour des années 1950 et qui, actuellement, distribue

12. «Germans in the East are still getting boost from Marshall Plan », Wall Street Journal, 26 janvier 1995, p. 1. 
environ la moitié des fonds affectés à un programme de financement des projets d'entreprises intitulé Programme européen de réhabilitation (ERP).

D'après le Wall Street Journal, l'impact de l'ERP sur le financement des PME dans l'ex-Allemagne de l'Est a été considérable.

En procurant du capital d'amorçage aux entreprises naissant dans le secteur privé, le Fonds de l'ERP a joué un rôle crucial dans la revitalisation de l'économie de la partie est de l'Allemagne et ce, après quatre décennies de planification centralisée communiste qui se sont révélées ruineuses. Les 26 milliards de dollars de crédits engagés depuis 1990 ont procuré des sources de financement peu coûteuses à plus de 150000 entreprises en phase de création, ont dopé l'investissement qui a dépassé les 60 milliards et ont permis de créer ou de sauvegarder 3 millions d'emplois. (Ibid.)

Une enquête récemment réalisée à l'initiative du ministère fédéral de l'Économie montre que la moitié des PME localisées dans l'ancienne Allemagne de l'Est ont reçu des crédits du Programme européen de réhabilitation. Les résultats indiquent que $40 \%$ des entreprises financées considèrent que les financements reçus se sont révélés d'une «importance vitale» et $10 \%$ jugent que si elles ont été capables de mettre en œuvre les investissements projetés, c'est grâce aux ressources financières obtenues dans le cadre de l'ERP.

\section{Dans quelle mesure le système de financement allemand est-il performant?}

\subsection{Contraintes de financement et de liquidité}

Que le système de financement allemand se révèle fort différent de ses homologues français, anglais, canadien ou américain est évident. En revanche, ce qui l'est moins concerne l'appréciation de l'efficience du système germanique en matière de financement des PME.

On sait, et la littérature consacrée à ce sujet ne manque pas de le souligner, que les marchés de capitaux présentent des modalités de fonctionnement qui leur sont spécifiques et qui les distinguent des marchés de produits et services. La particularité des marchés d'actifs financiers réside dans le niveau de risque accru associé aux demandes exprimées. Ce n'est pourtant que depuis fort peu de temps que l'attention des économistes se porte sur les conséquences de ce risque en ce qui concerne l'affectation de crédits - les marchés de capitaux ne sont pas systématiquement soldés au sens où l'offre serait égale à la demande pour tout niveau de prix donné. C'est ce qui a conduit Alan S. Blinder à préciser que, «il y a quelques années, en révisant la liste d'ouvrage à l'appui de mes enseignements, j'ai cherché des textes récents relatifs à la contrainte de liquidité et à l'investissement. Il n'y en avait point.» (1988, p. 196) 
Depuis la triste observation de Blinder (1988), une série d'études mettant en relation les contraintes de liquidité et les conditions de fonctionnement des marchés financiers ont été publiées. Dans ce domaine, Stiglitz et Weiss (1981) ont réalisé un apport capital en démontrant que la probabilité qu'a une entreprise de faire l'objet d'un rationnement de la part d'une banque n'est pas indépendante de sa taille. Au contraire, en tant que résultat de la sélection adverse qui s'exerce en situation d'asymétrie informationnelle, le risque de voir une firme subir un rationnement varie en raison inverse de sa taille.

C'est en fait à Fazzari, Hubbard et Petersen (1988) que l'on doit la présentation d'arguments définitifs à l'appui de la thèse selon laquelle la contrainte de liquidité tend à être de plus en plus marquée lorsque la taille de la firme diminue. De même, après avoir passé en revue les travaux empiriques consacrés à cette question, Chirinko conclut que, «alors que les preuves factuelles récemment exposées rendent compte de l'ampleur de la structure financière des firmes et des contraintes de liquidité qu'elles subissent, la question de leur origine et de leur sévérité demeure non résolue» (1993, p. 1904).

\subsection{Taille des firmes et contrainte de liquidité}

Cherchant à déterminer l'impact de la contrainte de financement sur la réalisation des investissements, Chirinko (1993, p. 1902) note que «la littérature consacrée à l'investissement a longtemps fait montre de schizophrénie en ce qui concerne le rôle des structures financières et des contraintes de liquidité». Comme l'indiquent Fazzari, Hubbard et Petersen (1988, p. 141),

[...] les modèles empiriques expliquant l'investissement industriel reposent généralement sur l'hypothèse de la firme représentative réagissant aux prix déterminés sur des marchés d'actifs centralisés. De fait, si toutes les entreprises avaient accès aux marchés de capitaux de manière équitable, les réponses des firmes aux variations du coût du capital ou aux inflexions des incitations fiscales à l'investissement ne différeraient qu'en raison d'écarts affectant la demande d'investissements.

Ainsi, la structure financière d'une entreprise ne constitue pas un facteur explicatif déterminant ses décisions en matière d'investissement à condition que l'unité considérée puisse substituer, sans avoir à supporter le moindre coût, des ressources externes aux capitaux internes. Si l'on suppose que les marchés de capitaux sont parfaits, alors les choix d'investissement spécifiques à la firme sont généralement indépendants des décisions financières prises.

L'hypothèse de marchés de capitaux parfaits a, bien évidemment, sérieusement été remise en cause. Et, dès lors que ce présupposé a été abandonné, il n'a plus été possible de considérer que les capitaux externes constituaient de parfaits substituts aux capitaux internes. L'une des principales conséquences 
de ce changement de point de vue réside dans l'influence qu'exercent la disponibilité des ressources propres, l'accès à l'endettement ou au financement par émission d'actions ainsi que d'autres facteurs financiers sur les décisions d'investissement.

Quel est le bon point de vue? Selon Fazzari, Hubbard et Petersen (1988, p. 142),

[...] les modèles conventionnels reposant sur la firme représentative dans lesquels la structure financière n'affecte pas la politique industrielle de l'entreprise peuvent correctement s'appliquer au cas des firmes en phase de maturité ayant des possibilités de développement clairement identifiées. En revanche, en ce qui concerne toutes les autres entreprises, les facteurs financiers semblent avoir une importance non négligeable au sens où les ressources externes ne sont pas parfaitement substituables aux fonds propres, tout particulièrement à court terme.

Trois raisons irréfutables rendent compte de la rigueur croissante avec laquelle s'exerce la contrainte de liquidité au fur et à mesure que la taille de la firme décroît. Stiglitz et Weiss (1981) indiquent que, à la différence de ce qui se passe sur la plupart des marchés, celui du crédit est particulier dans la mesure où le prix du bien échangé - le taux d'intérêt - n'atteint pas nécessairement un niveau permettant d'équilibrer l'offre et la demande. Ils expliquent ce phénomène en se référant à l'influence que le taux d'intérêt exerce non seulement sur le niveau de la demande exprimée par les emprunteurs potentiels, mais aussi sur le risque caractéristique des différents demandeurs. L'accroissement du taux d'intérêt s'accompagne alors d'une hausse du niveau de risque de chaque agent, cette relation conduisant les principaux offreurs de capitaux à décider rationnellement de limiter la quantité de crédits qu'ils distribuent au taux d'intérêt en vigueur.

La quantité d'information disponible relative à une entreprise n'est également pas indépendante de la taille de cette dernière. Au contraire, comme l'observent Petersen et Rajan (1992, p. 3),

[...] les firmes jeunes et de petite taille sont les plus fortement soumises au rationnement du crédit. La plupart des prêteurs potentiels ne disposent que de peu de renseignements sur les compétences en matière de gestion ou sur les opportunités d'investissement de telles entreprises, si bien qu'ils se trouvent dans l'incapacité de discriminer entre les différents niveaux de risque, de même qu'ils ne peuvent exercer de contrôle sur les investissements réalisés par l'emprunteur.

Si les prêteurs sont incapables d'apprécier la qualité ou le risque associé aux emprunteurs avec lesquels ils entrent en relation, un rationnement du crédit sera pratiqué, cette possibilité ayant fait l'objet d'une démonstration par Jaffee et Russel (1976). Ce phénomène ne va pas sans rappeler le problème des «rossignols» soulevé par George Akerlof (1970). L'asymétrie de l'information 
empêche en effet l'offreur de capitaux de s'engager dans un processus de discrimination par les prix séparant les emprunteurs en fonction de leurs niveaux de risque respectifs. Cependant, comme l'indique Diamond (1991), le risque associé à un crédit en particulier n'est pas indépendant de la durée de la relation entre la banque et l'emprunteur, notamment parce que l'information sur le risque sous-jacent caractéristique d'un client donné s'accumule au cours du temps. Grâce à l'expérience acquise, un prêteur se trouvera en mesure de caractériser le risque associé à la classe de clients à laquelle est rattaché un individu particulier.

Les grandes entreprises sont en mesure de financer leurs dépenses en capital par le biais de l'autofinancement, de l'émission d'actions ou grâce à la dette. Ce n'est pas le cas des PME qui rencontrent d'importantes limites tant en ce qui concerne le réinvestissement des bénéfices que leur capacité de procéder à des augmentations de capital par émission d'actions. Ainsi, Andrew et Eiseman (1984) montrent que le coût d'émission des créances non convertibles et des obligations proposées au public par le canal des courtiers et agents de change est passé de 2 à 5 millions de dollars et représente $1 \%$ des émissions d'un montant total supérieur à 50 millions de dollars. L'auteur note également que lorsque le montant des frais financiers est ajouté au coût d'émission, le coût des fonds levés peut dépasser les $30 \%$ au cours de la première année lorsque la firme est une PME.

Un nombre relativement imposant de recherches consacrées au cas américain et récemment publiées ont montré que l'impact exercé par la contrainte de liquidité est plus grand dans les petites entreprises que dans les grandes. Plus précisément, les PME apparaissent plus souvent incapables d'obtenir les financements au taux en vigueur sur le marché et, par conséquent, sont plus fortement touchées par le rationnement du crédit que les grandes entreprises. Fazzari, Hubbard et Petersen (1988) mettent l'accent sur le fait que les petites entreprises dont la structure juridique est de type société anonyme font face à des contraintes de liquidité et éprouvent d'énormes difficultés à obtenir les moyens de financement dont elles ont besoin au cours des périodes de récession économique. On peut donc considérer que la probabilité qu'une entreprise subisse une contrainte de liquidité diminue lorsque sa taille augmente.

Toujours d'après Fazzari, Hubbard et Petersen, les plus petites unités de production tendraient à être les plus étroitement dépendantes à la fois de la disponibilité des ressources internes et des crédits obtenus des banques que ne le sont les grandes entreprises. On est peu surpris de constater que les firmes de petite dimension s'adressent essentiellement aux banques lorsqu'elles éprouvent un besoin de financement dans le cadre d'un projet d'investissement. Toutefois, comme le souligne Stoll (1984), les PME doivent assumer 
un coût du crédit plus élevé que celui qui est facturé aux grandes entreprises. Citons, par exemple, une étude de la Réserve fédérale américaine sur les taux débiteurs pratiqués par les banques commerciales sur les crédits distribués entre le 3 et le 7 novembre 1986 qui montre que les crédits court terme à taux fixe étaient assortis d'un taux d'intérêt de $11,2 \%$ lorsque leur montant était inférieur à 24000 dollars. Ce taux baissait cependant rapidement et atteignait la valeur moyenne de $6,8 \%$ pour les crédits d'un montant supérieur à un million de dollars. Concernant les crédits à taux variable, le différentiel de taux s'est révélé moins important. Alors que le taux moyen des plus petits crédits s'élevait à $9,7 \%$, il était de $7,5 \%$ pour les prêts d'un montant élevé. Des structures de taux d'intérêt sensiblement équivalentes ont pu être repérées pour les prêts à long terme à taux fixe et à taux variable ${ }^{13}$. Les données chiffrées indiquent donc de manière très nette que le coût des ressources financières tend à diminuer lorsque le montant des crédits augmente.

Bien que, selon Fazzari, Hubbard et Petersen (1988), $99 \%$ des actions et $92 \%$ des obligations nouvellement émises soient réalisées par des grandes entreprises, ces dernières ne représentent que $74 \%$ du total des actifs industriels. Dans la mesure où les PME se révèlent essentiellement dépendantes de l'offre de crédits bancaires, elles sont plus soumises aux effets d'une restriction draconnienne du montant de prêts offerts, notamment au cours des périodes de récession économique. Ces mêmes auteurs mentionnent de nombreux éléments prouvant que les sources de financement dont disposent les PME tendent à se tarir plus rapidement pendant les phases de crise que celles auxquelles ont accès les entreprises de grande dimension, et cela au moins pour les États-Unis.

\subsection{L'étude d'Audretsch et Elston}

On ne dispose que de peu d'éléments permettant d'apprécier la sévérité de la contrainte de liquidité en Allemagne. Toutefois, une série de recherches récentes autorisent à penser que la structure financière allemande est telle qu'elle permettrait d'empêcher le développement d'un phénomène de rationnement (voir, par exemple, Cable, 1985).

Edwards et Fischer (1994) se sont interrogés sur l'impact exercé par la contrainte de liquidité subie par les firmes dans le cadre du système de financement allemand. De leur analyse résulte la proposition selon laquelle même si cette contrainte varie selon le type d'institutions financières propres à chaque pays, le modèle de financement allemand n'est pas fondamentalement différent d'un système de marché. Existent toutefois quelques éléments d'appréciation

13. Informations extraites de l'Office des PME des États-Unis, Rapport de 1987, tableau A2. 7, p. 91. 
sur l'intensité de la relation entre la rigueur de la contrainte de liquidité et la taille de l'entreprise, étudiée par Audretsch et Elston (1994) dans le cas de l'Allemagne.

Ce travail rend compte de la manière dont la contrainte financière est liée aux décisions d'investissement de la firme, la connexion entre ces deux facteurs étant réalisée grâce au ratio $Q$ de Tobin. Cet indicateur et la structure formelle dont il résulte ont permis de tester l'hypothèse selon laquelle les choix industriels et financiers de la firme seraient interdépendants lorsqu'est abandonnée l'hypothèse de marchés de capitaux parfaits. L'entreprise dont l'objectif consiste en la maximisation de sa valeur de marché pousse l'investissement jusqu'à ce que les bénéfices procurés par l'unité marginale de capital dépassent le coût marginal de l'investissement. La condition d'équilibre pour une firme se comportant de manière à maximiser son profit étant atteinte lorsque sa valeur de marché est égale au coût de remplacement du capital, la valeur de $\mathrm{Q}$ est nécessairement égale à l'unité.

L'un des principaux problèmes qui se posent lorsqu'on cherche à mesurer l'impact de la contrainte de liquidité sur l'investissement des entreprises discriminées en fonction de leur taille provient du manque de données fiables et adaptées à ce type de recherche. Pour leur étude empirique, Audretsch et Elston (1994) ont utilisé une base de données composée à partir de plusieurs rapports financiers d'entreprises industrielles allemandes cotées en Bourse sur une longue période. Ces auteurs n'ont trouvé aucun élément permettant d'étayer l'idée d'une protection du système financier allemand contre une éventuelle contrainte de liquidité. Au contraire, les résultats obtenus montrent que l'impact de la contrainte de financement sur l'investissement augmente systématiquement lorsque la taille des firmes diminue. Les plus petites entreprises seraient ainsi plus soumises à un risque d'insuffisance des ressources financières que les unités de grande taille, même dans un système caractérisé par des disparités entre les entreprises en fonction de leur dimension relativement faibles.

Audretsch et Elston (1994) démontrent cependant que l'organisation du système financier allemand a permis d'éviter qu'une contrainte de nature financière ne pèse sur les entreprises jusqu'au milieu des années 1970. Doit ici être mentionnée une caractéristique particulièrement frappante au cours de cette phase qui a été marquée par une abondance de crédit bon marché, cette période coïncidant cependant avec ce que d'aucuns ont appelé le miracle économique allemand. Depuis lors, rien ne permet de considérer que les entreprises nationales, et tout particulièrement les plus petites, n'aient pas été soumises à des contraintes de financement. 


\subsection{Le débat sur la politique économique}

La question de la viabilité du système de financement allemand, notamment en regard de sa capacité d'approvisionnement en fonds de la Mittelstand, a fait l'objet d'un débat fort intéressant. Comme l'indique le titre d'un article récemment paru dans la revue Business Week, «les Allemands se sont tout à coup mis à adorer haïr leurs banques», l'auteur écrivant que «s'est développé un fort ressentiment populaire à l'égard du pouvoir détenu par les banques». Ce qui au départ était une flambée est actuellement en train de devenir un véritable brasier où se déchaînent les passions politiques». Cabel, Griffin et Deeg confirment cette affirmation en soulignant que «le système de gestion des S.A. est actuellement en ruine» (1993, p. 15).

La caractéristique essentielle du système financier allemand est qu'il ne procure des ressources de manière efficiente que lorsqu'il s'est assuré de ce qui sera produit, par qui et à l'aide de quelles techniques de production. Par conséquent, les offreurs de fonds ont excellé dans la distribution de moyens de financement aux grandes compagnies appartenant à des secteurs traditionnels et aux moyennes entreprises bien implantées fabriquant des produits déjà éprouvés (Soskice, 1994). Il se révèle cependant nettement moins performant lorsqu'il s'agit d'affecter des fonds en dehors des branches traditionnelles, c'est-à-dire à l'ensemble des activités de haute technologie et innovantes. C'est d'ailleurs au niveau de ces secteurs naissants et en pleine expansion que le retard de l'Allemagne par rapport aux États-Unis et aux pays d'Asie du Sud-Est est le plus flagrant. Cela explique pourquoi une récente livraison de Newsweek (31 octobre 1994) était consacrée à la recherche des «raisons pour lesquelles l'Europe était en train de perdre la guerre des technologies». L'éditorial indiquait à ce propos que «le problème qui se posait à Siemens était loin d'être unique en son genre. Existent, au contraire, d'importants écarts de performances sur la plupart des produits de haute technologie européens, notamment ceux qui sont liés à la télématique, à savoir la mise en réseaux d'ordinateurs, les télécommunications, la télévision [...] Avec seules une poignée d'exceptions échappant à cette règle, la plupart des segments constitutifs des technologies de l'information se préparent à subir une déroute».

Un sentiment similaire se développe en Allemagne où l'on peut lire dans Der Spiegel que:

[...] le changement structurel qui s'est produit en Allemagne a exercé un impact sur l'économie inimaginable quelques années auparavant. La plupart des secteurs tels que l'automobile, la production de matériels d'équipement, la chimie et la fabrication de l'acier ne sont plus compétitifs sur les marchés mondiaux. De plus, les entreprises allemandes ne participent que faiblement aux activités du futur que sont les biotechnologies et l'électronique. (Der Spiegel, 1994, nº 5, p. 82-83) 
Enfin, Horst Albach avertit dans le Wall Street Journal du 29 avril 1994 que «lorsqu'on observe le secteur des microprocesseurs, on réalise qu'il se produit là un véritable désastre. Cela est également valable pour l'informatique et les technologies liées au secteur énergétique.»

Quel est le rapport entre l'incapacité de l'Allemagne à passer de ses domaines d'activités industrielles traditionnels aux technologies de pointe, aux nouvelles techniques de l'information et de la communication et le système de financement ? Comme le soulignait l'un des ténors du Parlement, une compagnie telle que Microsoft n'aurait pas eu une seule chance de naître en Allemagne, car les grandes banques nationales et les principales sociétés industrielles forment un cartel qui décourage les investissements des entrepreneurs innovants.

\section{Conclusion}

Le système de financement allemand se révèle fondamentalement différent de ceux que l'on peut observer aux États-Unis ou en Grande-Bretagne. Un mode d'organisation complexe des intermédiaires financiers a pu jusqu'à présent garantir que la Mittelstand soit approvisionnée en fonds plus généreusement que ne l'ont été les PME localisées dans les autres pays de l'OCDE. Il ne fait évidemment aucun doute qu'au cours de la période ayant suivi la Seconde Guerre mondiale, la Mittelstand a réalisé une contribution à la compétitivité de l'économie allemande bien plus grande que celle des PME implantées dans la plupart des autres pays industrialisés.

Cependant, en dépit de l'efficience du système de financement allemand, on dispose d'éléments permettant de considérer qu'il existe une relation positive entre les possibilités d'accès aux ressources financières et la taille des firmes. Cela tendrait à prouver que les PME allemandes, comme celles des autres pays, ont dû subir une contrainte de liquidité.

Le défi devant lequel est placé le système financier allemand doit toutefois être rapidement relevé; il s'agit en effet de savoir comment le faire évoluer de façon à faciliter le financement des jeunes entreprises dans les secteurs innovants. En d'autres termes, même si les modalités d'affectation des ressources prêtables existantes se sont révélées parfaitement efficientes lorsqu'il s'agissait d'affecter des ressources aux moyennes entreprises et aux grandes firmes intervenant dans des secteurs traditionnels, il n'est pas dit que ces dispositions suffisent à garantir l'octroi de moyens financiers aux entreprises n'appartenant pas à ce groupe. 


\section{Bibliographie}

ACs, Z.J. et D.B. Audretsch (éd.) (1993), Small Firms and Entrepreneurship: An East-West Perspective, Cambridge, Cambridge University Press.

AKERLOFF, G.A. (1970), "The market for 'Lemons' : quality uncertainty and the market mechanism», Quarterly Journal of Economics, vol. 84, p. 488-500.

ALBACH, H. (1984), «Finanzierung mittelständischer unternehmen», Institut für Mittelstandsforschung, Neue Folge, $\mathrm{n}^{\circ} 8$.

ANDREWS, L.V. et C.P. EISEMANN (1984), "Who finances small business in the 1980s? ", dans P.M. Horvitz et R.R. Pettit (éd.), Small Business Finance: Problems in the Financing of Small Business, Greenwich, Conn., JAI Press, p. 75-96.

AUDRETSCH, D.B. (1989), «Legalized cartels in West Germany », Antitrust Bulletin, vol. $73, \mathrm{n}^{0} 3$, p. $579-600$.

AudretsCh, D.B. et J.A. Elston (1994), «Does firm size matter? Evidence on the impacts of liquidity constraints on firm investment behavior in Germany », Cahier de recherche, $\mathrm{n}^{\circ}$ 1072, novembre, Centre for Economic Policy Research (CEPR).

BANNASCH, H.G. (1993), "The evolution of small business in East Germany », dans Z.J. Acs et D.B. Audretsch (éd.), Small Firms and Entrepreneurship : An East-West Perspective, Cambridge, Cambridge University Press, p. 182-189.

BLINDER, A.S. (1988), «Financing constraints and corporate investment: discussion and comments", Brookings Papers on Economic Activity, p. 196-200.

BUNDESMINISTER FÜR FORSCHUNG UND TECHNOLOGIE (1982), «Die messung wissenschaftlicher und technischer Tätigkeiten», Bonn Bundesminister für Forschung und Technologie.

BUNDESMINISTER FÜR FORSCHUNG UND TECHNOLOGIE (1985), «Statistische Informationen», Bonn.

BUNDESMINISTER FÜR WIRTSCHAFT (1987), «Bericht des Bundeskartellamtes über seine Tätigkeit in den Jahren 1985 / 1986 sowie über Lage und Entwicklung auf seinem Aufgabengebiet» ( $\$ 50$ GWB), Bonn, Bundestagsdrucksache.

CABLE, J. (1985), «Capital market information and industrial performance: the role of West German banks », Economic Journal, vol. 95, p. 118-32.

CABEL, C.F., J.R. GRIFFIN et R.E. DEEG (1993), «Making money talk: towards a new debtor-creditor relation in German banking», Communication présentée à la Conférence on Relational Investing, Center for Law and Economic Studies, Columbia University School of Law, 6-7 mai, New York.

CHIRINKo, R. (1993), «Business fixed investment spending: modeling strategies, empirical results and policy implications ", Journal of Economic Literature, vol. 31 , p. 1875-1911. 
DEEG, R. (1991), «The state, banks and economic governance in Germany », German Politics, vol. 2, $\mathrm{n}^{\circ}$ 2, p. 149-176.

DEEG, R. (1992), «Banks and the state in Germany: the critical role of subnational institutions in economic governance», Thèse de doctorat non publiée, MIT.

DEUTSCHE BUNDESBANK (1991a), «The profitability of German banks in 1990 », Rapport mensuel de la Deutsche Bundesbank, août.

DEUTSCHE BUNDESBANK (199lb), «The significance of shares as financing instruments », Rapport mensuel de la Deutsche Bundesbank, octobre.

DIAMOND, D. (1991), «Monitoring and reputation: the choice between bank loans and directly placed debt », Journal of Political Economy, vol. 99, p. 688-721.

EDWARDS, J.S. et K. FISCHER (1994), The German Financial System, Cambridge, Cambridge University Press.

FAzZARI, S., R.G. Hubbard et B.C. Petersen (1988), «Financing constraints and corporate investment », Brookings Papers on Economic Activity, p. 141-206.

FRITSCH, M. (1993), "The role of small firms in West Germany », dans Z.J. Acs et D.B. Audretsch (éd.), Small Firms and Entrepreneurship: An East-West Perspective, Cambridge, Cambridge University Press, p. 38-54.

HUNSDIEK, D. et E. MARY-STROBL (1987), «Grundungsfinanzierung durch den staatfacten, erfolg und wirkung», Institut fur Mittelständsforschung, Neue Folge, $n^{0} 17$.

JAFFEE, D.M. et T. RUSSELL (1976), «Imperfect information, uncertainty and credit rationing», Quarterly Journal of Economics, vol. 90, p. 651-666.

Levratto, N. (1994), «Small firm finance in France», Small Business Economics.

LOVEMAN, G. et W. SENGENBERGER (1991), «The re-emergence of small scale production: an international comparison», Small Business Economics, vol. 3, $n^{0} 1$, p. 1-38.

MAYER, C. et I. AleXANDER (1990), «Banks and securities markets: corporate financing in Germany and the United Kingdom », Journal of the Japanese and International Economies, vol. 4, p. 181-194.

PETERSEN, M.A. et R.G. RAJAN (1992), «The benefits of firm-creditor relationships : evidence from small business data», Cahier de recherche, $n^{0} 362$, University of Chicago.

SCHWALBACH, J. (1989), «Small business in German manufacturing », Small Business Economics, vol. 1, n² 2, p. 129-136.

SiMON, H. (1990), «Hidden champions », Zeitschrift für Betriebswirtschaft, vol. 60, $n^{\circ} 9$, p. 875-890. 
SOSKICE, D. (1994), «Innovation strategies of companies: a comparative institutional approach of some cross-country differences», dans M. Dierkes et W. Zapf (éd.), Institutionenvergleich und Institutionendynamik, Berlin, Édition Sigma, p. 271-289.

STIGLITZ, J. et A. WeISS (1981), «Credit rationing in markets with imperfect information", American Economic Review, vol. 71, p. 393-410.

StockmanN, R. et R. Leicht (1994), "The pattern of changes in the long term development of establishment size», Small Business Economics, vol. 6, n ${ }^{0}$ 6, p. 451-464.

STOLL, H.R. (1934), «Small firms' access to public equity financing», dans P.M. Horvitz et R.R. Pettit (éd.), Small Business Finance: Problems in the Financing of Small Business, Greenwich, Conn., JAI Press, p. 187-238.

VITOLS, S.I. (1994), «German banks and the modernization of the small firm sector: long term finance in comparative perspective», texte non publié, Wissenschaftszentrum Berlin für Sozialforschung. 